\title{
Dysphagia management during COVID-19 pandemic: A review of the literature and international guidelines
}

\author{
Sibel Eyigör ${ }^{1}$, Ebru Umay²® \\ Department of Physical Medicine and Rehabilitation, Ege University Faculty of Medicine, Izmir, Turkey \\ Department of Physical Medicine and Rehabilitation, Dışkapı Ylldırım Beyazıt Training and Research Hospital, Ankara, Turkey
}

Received: February 17, 2021 Accepted: March 20, 2021 Published online: September 01, 2021

\begin{abstract}
In this review, we present the safest and most effective diagnosis and treatment approaches to dysphagia during the novel coronavirus-2019 (COVID-19) pandemic in the light of available data, relevant literature, and personal experiences. Evaluations for dysphagia patients should be based on clinical assessment during the COVID-19 pandemic and instrumental assessment should be planned for very few number of patients. The main approach to rehabilitation must depend on compensatory methods, texture-modified foods, and postural strategies. Direct treatment methods should be avoided and home-based exercise programs should be encouraged. It is also obvious that there is a need for the development of new strategies for telemedicine/telerehabilitation practices in the new world order.
\end{abstract}

Keywords: COVID-19, dysphagia, management, swallowing, treatment.

Since the World Health Organization (WHO) declared the novel coronavirus-2019 (COVID-19) pandemic, many disciplines concerning swallowing disorders, similar to all other health professionals from different fields, have faced problems about the actions to be taken during the process. Guidelines have been developed on dysphagia and in other fields to assist experts in clinical practice. In this regard, an updated road map has been prepared according to the conditions of our country, taking into account the literature and clinical practice.

\section{COVID-19 AND DYSPHAGIA RELATIONSHIP}

Severe acute respiratory syndrome-coronavirus-2 (SARS-CoV-2) responsible for COVID-19 enters into the host cells via the angiotensin-converting enzyme 2 (ACE-2) receptor in human lower respiratory tract. The ACE-2, which is primarily associated with cardiovascular diseases, is a membrane protein expressed in the lungs, heart, kidneys and intestines, leading to disorders mainly in the pulmonary system, as well as the cardiovascular, gastrointestinal and hepatic systems. ${ }^{[1]}$

Unfortunately, all the conditions and procedures implemented in connection with COVID-19 itself or its treatment such as acute respiratory distress syndrome (ARDS), multiple organ failure, sepsis, neurological complications, loss of smell and taste, sedative agents used in the intensive care, non-invasive mechanical ventilation, oxygen treatment, intubation, tracheostomy, malnutrition and muscle wasting affect swallowing functions negatively. ${ }^{[2]}$ One study conducted in Turkey showed that $20.6 \%$ of COVID-19 patients suffered from dysphagia. ${ }^{[3]}$ However, this ratio is considered to increase with further studies to be carried out. In this review, we discuss the relationship between COVID-19 and dysphagia from different aspects in the first place.

\footnotetext{
Corresponding author: Sibel Eyigör, MD. Ege Üniversitesi Tıp Fakültesi, Fiziksel Tıp ve Rehabilitasyon Anabilim Dalı, 35100 Bornova, İzmir, Türkiye. e-mail: eyigor@hotmail.com 


\section{PATIENT CHARACTERISTICS}

Although new information is obtained about COVID-19 every day, studies defining the risk factors and risk groups are still insufficient. Studies define such characteristics as old age, presence of additional comorbidities, smoking and being a healthcare professional as risk factors and associate them with severe disease. ${ }^{[4-6]}$

\section{Old age}

In studies including COVID-19 patients, the mortality rate is 18 times higher among patients aged $\geq 60$ years and approximately 70 times higher among those aged $\geq 80$ years. ${ }^{[7,8]}$ The number and size of muscle fibers start to decline with advancing age all over the body, and the increase in muscle loss leads to functional disorders. Sarcopenia, which is characterized by the degenerative loss of muscle mass and strength, is almost an unchangeable condition related to aging. In addition, respiratory muscles get weaker and lose their elasticity, lowering both perfusion and ventilation capacities. Sphincter muscles get weaker and acid secretion decreases in the stomach. Nerve conduction velocities slow down the reflexes. Losses of sensory functions including taste and smell are also among common problems. Changes in the immune system called immunosenescence brings about susceptibility to infection, autoimmunity, and malignity. ${ }^{[9]}$ Such changes cause COVID-19 to have a more severe progression in elderly.

As in all other systems, changes also occur in swallowing functions with aging. Presbyphagia is characterized by the changes in the swallowing mechanism of healthy older adults occurring with the normal aging process. Each phase of deglutition is affected. Changes seen in presbyphagia include bolus control, preparation and damage in its transportation, delayed triggering and initiation of swallowing, delayed opening of the upper esophageal sphincter and weak esophageal peristalsis. ${ }^{[10]}$

Recent studies have reported that healthy older adults have dysphagia that they are not aware of and, thus, they experience varying degrees of dysphagia in their daily lives. ${ }^{[1]}$ Hence, together with these disorders, which are present at the background, COVID-19 may lead to dysphagia development or increase the severity of existing dysphagia.

\section{Comorbidity}

In studies conducted with COVID-19 patients, fatality rates are reported as $0.9 \%$ in patients with no comorbidities, $10.5 \%$ in the presence of a cardiovascular disease, $7.3 \%$ in the presence of diabetes mellitus and chronic obstructive pulmonary disease (COPD), and $6 \%$ in patients with hypertension. ${ }^{[12]}$ Hypercoagulability and metabolic acidosis that develop in the aforementioned diseases may cause mortality.

Cardiovascular, metabolic/endocrine and respiratory diseases are potential, albeit indirect, risk factors for oropharyngeal dysphagia. According to related studies, the fact that these disease groups cause cerebrovascular diseases is a risk factor for the development of dysphagia. ${ }^{[13]}$ In addition, in cases of COPD, asthma, pulmonary fibrosis, and heart failure, leading to impaired swallowing synchronization, dysphagia and aspiration risk increases. ${ }^{[14,15]}$

\section{Smoking}

While the initial findings about the relationship between smoking and COVID-19 were controversial, a recent meta-analysis have shown that there is a relationship between active cigarette smoking and the disease. ${ }^{[16]}$ The smoking-related decline in the lung capacity and chronic inflammation have been reported to accelerate the lung damage caused by the disease.

Since it inhibits the pharyngeal swallowing reflex and cough reflex, has a direct toxic effect on the ciliary mucus of the respiratory tract, and due to its carcinogenic components, cigarette smoking itself is a risk factor for dysphagia. ${ }^{[17]}$

\section{CHARACTERISTICS OF COVID-19}

Based on the latest data from the WHO, the number of COVID-19 cases worldwide is approximately 110 million of as of February 2021. ${ }^{[18]}$ Since COVID-19 was accepted as a global pandemic, the hospitalization rate was reported as 380.3 per 100,000 population. ${ }^{[12]}$ As the methods to combat the disease improved, the high rates at the breakout fell to 17.8 per 100,000 population as of December 2020. Hospitalization in COVID-19 is usually associated with ARDS occurring due to pneumonia, pulmonary or non-pulmonary reasons.

\section{Tachypnea and dyspnea}

Tachypnea and dyspnea are frequent symptoms observed in COVID-19 patients. The increased respiratory rate ( $>25$ breaths/min) and disrupted breathing-swallowing coordination increase the risk of aspiration for both healthy persons and those at risk for dysphagia. ${ }^{[19]}$ This desynchronization becomes more apparent with the disruption of ventilation due 
to the alveolar damage and edema and of the perfusion due to hypercoagulability caused by COVID-19, leading to aspiration. ${ }^{[20]}$ Studies have reported that aspiration occurs, particularly with large volumes $(100 \mathrm{~mL})$ of liquids in patients with disordered breathing patterns. ${ }^{[19]}$ Besides tachypnea and dyspnea, low oxygen saturation ( $\mathrm{SpO} 2)$ is not always associated with aspiration and $<94 \% \mathrm{SpO}_{2}$ is defined as an increased risk for aspiration by relevant studies. ${ }^{[21]}$

\section{Loss of taste}

Taste and smell play an important role in the selection of diet, metabolism, eating habits, and quality of life. Studies have shown that taste/smell dysfunction is among the most common symptoms observed in COVID-19 patients. ${ }^{[22]}$ The prevalence of taste alterations ranges between 11 and $88.8 \%$. As a matter of fact, sudden-onset ageusia (loss of taste) and anosmia (loss of smell) is recognized as the key clinical symptoms of COVID-19. ${ }^{[23]}$ Possible mechanisms for this symptom include a direct damage of the olfactory and gustatory receptors, damage of the ACE- 2 receptor in the oral cavity, and decreased interleukin (IL)-6 levels. Improvement is seen 7 to 14 days after the infection, while it may be persistent in some patients. The mechanism responsible for its persistence has not been clearly known, yet. When persistent, it is considered that dysphagia and nutrition problems may be faced in the long-term. Moreover, disruptions in the consumption of salt, sugar, and fat and over consumption leads to the deterioration of accompanying diseases, such as hypertension and diabetes. Thus, nutritional monitoring is recommended for patients with COVID-19-related loss of taste/smell. ${ }^{[24]}$ Therefore, considering the relationship between loss of taste/smell and swallowing disorder, there is a clear need to pay more attention to the issue..$^{[25-27]}$

\section{Neurological findings}

Recent studies have reported neurological findings in COVID-19 patients with moderate-tosevere pneumonia. ${ }^{[28,29]}$ The disturbances in cognitive functions and consciousness defined as delirium develops as a result of a direct infection of the central nervous system, hyperinflammatory cytokine storm, increased oxidative stress, and hypoxemia. These disturbances in the cognitive function affect all the phases of swallowing increasing the risk for aspiration. ${ }^{[28]}$ Similarly, dysphagia may become a critical problem in cases manifested with stroke or Guillain-Barré syndrome. ${ }^{[2]}$
There is an ongoing discussion on the fact that the virus targets the cerebral cortex, subcortex and brainstem structures, peripheral nerves and muscles, as well as the complete swallowing network. These are the hypotheses that the virus is neurotrophic and affects the cranial nerves (vagus, hypoglosus) involved in swallowing and about the effect of the virus on the sensitivity of the pharynx and larynx..$^{[26,30]}$

\section{Malnutrition}

The relationship of nutrition with muscle mass and dysphagia is discussed in many diseases primarily geriatric syndromes. ${ }^{[31]}$ The elevated doses of sedatives and opioids during intensive care have been found to contribute to intestinal dysmotility, weight loss, and cachexia in COVID-19 patients. Moreover, the cytokine storm in COVID-19 is associated with muscle mass loss, weakness, and fatigue. ${ }^{[32]}$ Swallowing problems are listed among the determining factors for malnutrition. ${ }^{[30,33]}$ Malnutrition and muscle wasting seen in COVID-19 patients contribute to the development of dysphagia. ${ }^{[31]}$ All types of potential muscle losses and nutritional disorders to develop in COVID-19 patients are intertwined with many clinical conditions mentioned in this section and pose a risk for dysphagia.

\section{CHARACTERISTICS OF COVID-19 TREATMENT}

High-flow oxygen therapy (nasal high-flow oxygen therapy- $\mathrm{HHO}_{2}$ )

Nasal $\mathrm{HHO}_{2}$ used in the treatment of hypoxemia secondary to lung involvement in COVID-19 patients is an effective method which is more reliable than interventions of invasive ventilation. ${ }^{[34,35]}$ The $\mathrm{HHO}_{2}$ improves patient comfort, generates a continuous positive airway pressure effect, decreases the resistance in respiratory tracts, and improves secretion clearance. However, this stable positive pressure affects the chemo- and mechanoreceptors in the pharynx and larynx causing a delayed swallowing reflex, hypoesthesia in these areas, laryngeal adduction, a weakened retching and cough reflex, and a disrupted respiratory-swallowing pattern. ${ }^{[36,37]}$

\section{ARDS, intensive care and endotracheal intubation}

In COVID-19, patients who are resistant to oxygen supportive therapy and develop ARDS are required to be transferred to the intensive care unit (ICU) and intubated. Intensive care, itself, is a risk factor for dysphagia. In addition to the muscle and nerve 
injuries due to the direct trauma of orotracheal and tracheostomy tubes to the vocal cord, tongue base, epiglottis, arytenoids, and the local effects such as sensory losses caused by edema and lower input, critical illness neuromyopathy, diffuse atrophy due to the general sedation-related muscle immobilization throughout the body pave the way for the development of dysphagia, as well. ${ }^{[38,39]}$

Intubation in the ICU and extended mechanical ventilation are the most important independent risk factors for dysphagia. ${ }^{[36,40]}$ Local mucosal injuries that occur due to endotracheal intubation, muscle atrophy, and sensory loss cause the development of silent micro-aspirations. Studies conducted in ICUs report that 83 to $94 \%$ of laryngeal injuries are seen after the average one-week intubation and that half of the patients develop resulting dysphagia. ${ }^{[11,42]}$ It has been also demonstrated that this condition usually continues for nearly one week following extubation, while it may be prolonged up to one to three months. ${ }^{[43]}$ Each intubation of two days or longer is stated as a risk factor for chronic dysphagia. ${ }^{[4]}$ The first 24 -h post-extubation is defined as the most critical period, when silent aspirations are the most frequent. ${ }^{[43]}$ The risk of saliva and secretion aspiration increases in the prolonged prone position implemented in the follow-up of COVID-19 ARDS, which causes difficulty in terms of oral hygiene. ${ }^{[36]}$ Similarly, implementation of a tracheostomy increases the risk for dysphagia and aspiration in this patient group. The presence of a tracheostomy leads to laryngeal and pharyngeal dysfunction during swallowing in $87 \%$ of cases. ${ }^{[45,46]}$

Dysphagia-related tracheostomy complications include reduced laryngeal activity, muscle atrophy, vocal cord paralysis, esophagus obstruction, and development of tracheoesophageal fistula due to the reduced coughing reflex and swallowing frequency and inflated cuff as a result of the laryngopharyngeal sensory loss, glottal closure insufficiency and subglottal pressure decrease depending on the duration and volume of the tracheostomy and cuff inflation pressure. ${ }^{[45,47]}$

\section{Drugs used in the ICU setting}

Sedative agents and opioids used in the ICU are associated with an increased risk of neuromuscular muscle weakness, disruption of the pharyngeal and/or lingual propulsion of the bolus and stasis, pharyngeal dysfunction, and breathing-swallowing coordination disturbance. ${ }^{[2,26]}$ There is also an increased possibility of gastroesophageal reflux due to the lying position and sedative agents. ${ }^{[26]}$ The presence of reflux is considered as a risk factor for dysphagia and aspiration. ${ }^{[48-50]}$

To sum up, factors that lead to a severe progression of COVID-19, changes caused by the disease in the body and treatments implemented may all be risk factors for dysphagia. The possibility of dysphagia development should be kept in mind for COVID-19 patients, particularly for those hospitalized with a severe progression of the disease. Unfortunately, clinicians do not have a sufficient level of awareness of the subject, yet. ${ }^{[30,51]}$

\section{DYSPHAGIA MANAGEMENT DURING THE COVID-19 PANDEMIC}

Methods involving the diagnosis and treatment of dysphagia include evaluations concerning the fact that the risk of infection of the virus is considerably high. The most common questions that clinicians ask in the clinical practice are how to approach dysphagia patients during the COVID-19 pandemic and what needs to be considered in requiring further examination and treatment. Recommendations for approaches to be used in the clinical practice for COVID-19 are made in different fields by different disciplines and different countries. ${ }^{[2,52-59]}$ The most prominent of all these concerns is the use of personal protective equipment (PPE) by the healthcare staff. Protection of the clinician has to come first.

Although a specific strategy has not yet been developed as to which patient to examine, examination is recommended for patients who are intubated for more than $48 \mathrm{~h}$, particularly for COVID-19 patients. It is suggested that the examination is performed by a nurse or speech language pathologist. ${ }^{[40]}$ Bedside assessment is recommended rather than a detailed instrumental examination. Variable examination timepoints are recommended (e.g., one to five days after extubation). Elective and non-urgent cases could be deferred. Assessment should be restricted to patients with a serious condition such as aspiration pneumonia, malnutrition or sepsis, those having dysphagia of unknown origin or a life-threatening disease such as head-neck cancer or neuromuscular disorders or those who are unable to take sufficient nutrition. ${ }^{[52]}$

In addition, methods such as the screening tests can be used asking questions to the patient or his/relatives. ${ }^{[60]}$ Non-invasive interventions should be the first choice as far as possible -including a detailed medical history, Eating Assessment Tool-10 (EAT-10) for dysphagia symptoms), and cranial nerve 
examination. ${ }^{[59,61,62]}$ The use of such tools as Toronto Bedside Swallowing Screening Test (TOR-BSST), Yale Swallow Protocol, Volume Viscosity Swallow Test (V-VST), and Mann Assessment of Swallowing Ability (MASA) is recommended for screening oropharyngeal dysphagia; however, it must be remembered that swallowing screening has not yet been validated for COVID-19 patients. ${ }^{[54]}$

\section{Clinical examination}

It is advocated that each patient must be considered a potential COVID-19 patient, unless proven otherwise. ${ }^{[63]}$ It is recommended that sufficient information should be obtained about the patient, and such assessments should be made by a multidisciplinary team and only conscious patients with a stable respiratory status should be assessed. ${ }^{[2]}$

A particular attention must be paid to the use of aerosol-generating procedures and the donning/doffing PPE procedure. The donning of PPE (N95 or FFP2-3 masks, gown, face shield/goggles and gloves, bone with $\operatorname{COVID}(+)$ patients; surgical masks, goggles, face shields and gloves with COVID(-) patients) is considered mandatory ${ }^{[2,52]}$ Hand hygiene is strongly recommended before and after contact with patients and after doffing off the PE. ${ }^{[59]}$

Practitioners should be positioned at a 1 to $2-\mathrm{m}$ distance to the side of the patient, instead of face-to-face examination. Gag reflex and voluntary control of coughing are not recommended. It is suggested that orofacial muscle and cranial nerve examinations should be performed, while speaking and resting, and oral cavity examinations should be avoided and the use of light is necessary. Assessment through self-feeding is recommended. Examination times should be limited to a maximum duration of 10 to 15 min keeping the number of repeated swallows low. Vocal quality, aspiration signs, oral stasis-residue, triggering of the swallow response, laryngeal and hyoid movements must be monitored by maintaining follow-ups from a distance from the patient. Laryngeal palpation and hyoid movement assessments are advised not to be performed according to a majority of authorities. ${ }^{[2]}$

\section{Instrumental examination}

Evaluations must be based on clinical assessment. $^{[2]}$ Such practices as flexible fiberoptic endoscopic evaluation of swallowing (FEES), videofluoroscopy (VF), pharyngoesophageal manometry, and electroneuromyography (ENMG) should be performed only in case of life-threatening conditions. They should be planned, only if clinical assessment is not sufficient for treatment or if the clinical condition of the patient is not appropriate for postponing assessment. It must be guaranteed that all persons present in the environment of practices and those using the same instrument are COVID-19 free. ${ }^{[52]}$

Instrumental assessment is recommended for a very limited group. ${ }^{[40]}$ It must be planned by a multidisciplinary team only in the presence of silent aspiration and excessive secretion, insufficient clinical evaluation, risk of dysphagia-related dehydration, malnutrition or aspiration, and if the intervention cannot be deferred or alternative nutrition cannot be provided. ${ }^{[40]}$ Due to working in a short distance and the risk of coughing-sneezing during the procedure, FEES implementation poses the clinician at high risk for viral load. ${ }^{[52,54]}$

Aerosol-generating procedures and donning/ doffing PPE procedures must be followed under very strict conditions of protection. Instrumental assessment should be made by a minimum number of healthcare providers, as possible. It should be planned in the ICU, in a negative pressure isolation room with air filter, but not face-to-face and using disposable fluoroscopy, if available. The room must be disinfected afterwards. Laryngeal sensitivity test is contraindicated in these patients. Two different viscosity-consistency and maximum two trials are recommended in the FEES protocol. Standardized scales such as the Penetration Aspiration Scale (PAS) and Yale Residue Scale (YRS) should be used..$^{[2]}$ Endoscopy must be removed carefully to avoid coughing and sneezing. The primary physician must be informed in case of a laryngopharyngeal reflux or excessive secretion. ${ }^{[52]}$

The VF is considered to be a relatively safe procedure. However, the areas, corridors, and elevators through which the patient passess while being transferred to the procedure room pose a risk for contamination. Recommendations are made for the disinfection of the environment, presence of an air filter, and a low number of healthcare providers inside. Sufficient time must be allocated for cleaning between implementations. Indications of VF implementation are similar to FEES, while it must be performed in only a limited number of cases. Similar recommendations are made for pharyngoesophageal manometry, as well.

\section{Rehabilitation}

Considering the recommendations of international societies of dysphagia, variances among the duration, frequency, and environment of treatment in dysphagia rehabilitation attract attention. ${ }^{[52,54,59,64]}$ 
The use of compensatory rehabilitation methods is advocated. It is recommended that durations of treatment should be shortened. Risky approaches in terms of virus load such as thermal tactile simulation and expiratory and inspiratory muscle strength training must be avoided. Similarly, face-to-face breathing exercises by the patient and therapist and special swallowing maneuvers that require coughing are not recommended. ${ }^{[52]}$ On the other hand, tongue, lips, neck, and temporomandibular joint range of motion and tongue, lips, neck and temporomandibular joint strengthening exercises, and Shaker exercises are recommended due to the low infection risk they pose. ${ }^{[59]}$

Patient and their caregivers should be informed about following oral hygiene directions..$^{[2,52]}$ Since chlorhexidine frequently used in clinical practices is not effective in COVID-19, $0.2 \%$ povidone or $1 \%$ hydrogen peroxide can be used for mouth washing/rinsing. ${ }^{[59]}$

After patient information and teaching the exercises, aerosol-generating procedures and donning/doffing PPE procedures must be complied (both by following the rules during education and cleaning around after the patient). Recommendations are made in a well-ventilated room, being physically distant and wearing masks, and the presence of the patient and his/her attendant only, if possible. All the procedures must be completed as soon as possible (10 to $15 \mathrm{~min}$ ).

It is advocated that treatments such as electric stimulation and transcranial magnetic stimulation should be deferred. ${ }^{[2]}$ However, recommendations of the Society of Swallowing and Dysphagia of Japan (SSDJ) include the use of electric stimulation therapy. ${ }^{[59]}$

Non-oral nutrition is recommended in the presence of cough after swallowing liquid and thickened liquid, while nutrition with thickened fluids is recommended in the presence of cough with liquids only. A nasogastric (NG) tube or percutaneous endoscopic gastrostomy (PEG) is required, if oral nutrition is not safe. The latter is preferred to NG owing to its simplicity of insertion and the reduced risk to both patient and healthcare providers. ${ }^{[40]}$

Telepractice-telemedicine and telehealth consultation methods are frequently encountered among the recommendations made by the societies and organizations related to dysphagia treatment in the literature. Telemedicine consultation systems have been reported to be effective in patients who develop dysphagia during the COVID-19 pandemic. ${ }^{[65]}$ The system is particularly recommended for the diagnosis and follow-up of patients who have neurological disorders including stroke and head and neck cancer in which dysphagia is frequently seen. ${ }^{[2,52,53]}$

However, the use of telemedicine methods requires certain criteria. ${ }^{[53]}$ It is reported to be suitable for patients, who have the cognitive capacity to understand the system, be positioned in front of a camera and maintain this position for a while, and hear, see and comprehend visual/audial stimulants and who have a family/caregiver support. ${ }^{[53]}$

In concussion, this review provides a brief summary of the safest and most effective diagnosis and treatment approaches to dysphagia during the COVID-19 pandemic in practical terms based on the available data, relevant literature, and personal experiences. Considering the viral mutations, absence of an effective treatment for COVID-19, and difficulties to reach the vaccines, it is evident that we must follow such recommendations for a while. Evaluations for dysphagia patients must be based on clinical assessment during the COVID-19 pandemic and instrumental assessment should be planned for very few number of patients. The main approach to rehabilitation must depend on compensatory methods, texture-modified foods and postural strategies. Direct treatment methods should be avoided and home-based exercise programs should be encouraged. It is also obvious that there is a need for the development of new strategies for telemedicine/telerehabilitation practices in the new world order.

\section{Declaration of conflicting interests}

The authors declared no conflicts of interest with respect to the authorship and/or publication of this article.

Funding

The authors received no financial support for the research and/or authorship of this article.

\section{REFERENCES}

1. Wu D, Wu T, Liu Q, Yang Z. The SARS-CoV-2 outbreak: What we know. Int J Infect Dis 2020;94:44-8.

2. Vergara J, Skoretz SA, Brodsky MB, Miles A, Langmore SE, Wallace S, et al. Assessment, Diagnosis, and Treatment of Dysphagia in Patients Infected With SARS-CoV-2: A Review of the Literature and International Guidelines. Am J Speech Lang Pathol 2020;29:2242-53.

3. Özçelik Korkmaz M, Eğilmez OK, Özçelik MA, Güven M. Otolaryngological manifestations of hospitalised patients with confirmed COVID-19 infection. Eur Arch Otorhinolaryngol 2020:1-11. 
4. Zhou F, Yu T, Du R, Fan G, Liu Y, Liu Z, et al. Clinical course and risk factors for mortality of adult inpatients with COVID-19 in Wuhan, China: a retrospective cohort study. Lancet 2020;395:1054-62.

5. Emami A, Javanmardi F, Pirbonyeh N, Akbari A. Prevalence of underlying diseases in hospitalized patients with COVID-19: A systematic review and meta-analysis. Arch Acad Emerg Med 2020;8:e35.

6. Liu W, Tao ZW, Wang L, Yuan ML, Liu K, Zhou L, et al. Analysis of factors associated with disease outcomes in hospitalized patients with 2019 novel coronavirus disease. Chin Med J (Engl) 2020;133:1032-8.

7. Sahu KK, Mishra AK, Lal A. COVID-2019: Update on epidemiology, disease spread and management. Monaldi Arch Chest Dis 2020;90.

8. Jin Y, Yang H, Ji W, Wu W, Chen S, Zhang W, et al. Virology, epidemiology, pathogenesis, and control of COVID-19. Viruses 2020;12:372.

9. Güleç M. Old age and immune system. Turkiye Klinikleri J Geriatr-Special Topics 2015;1:1-7.

10. Butler SG, Stuart A, Leng X, Wilhelm E, Rees C, Williamson J, et al. The relationship of aspiration status with tongue and handgrip strength in healthy older adults. J Gerontol A Biol Sci Med Sci 2011;66:452-8.

11. Umay E, Eyigor S, Karahan AK, Keskin D, Karaca G, Unlu $\mathrm{Z}$, et al. Which swallowing difficulty of food consistency is best predictor for oropharyngeal dysphagia risk in older person? European Geriatric Medicine 2019;10:609-17.

12. European Centre for Disease Prevention and Control. 2020. Available at: https://covid19-surveillance-report.ecdc. europa.eu [Accessed: April 17, 2021]

13. Almeida TM, Gomes LMS, Afonso D, Magnoni D, Mota ICP, França JÍD, et al. Risk factors for oropharyngeal dysphagia in cardiovascular diseases. J Appl Oral Sci 2020;28:e20190489.

14. Lin TF, Shune S. Chronic obstructive pulmonary disease and dysphagia: A synergistic review. Geriatrics (Basel) 2020;5:45.

15. Cvejic L, Bardin PG. Swallow and aspiration in chronic obstructive pulmonary disease. Am J Respir Crit Care Med 2018;198:1122-9.

16. Gülsen A, Yigitbas BA, Uslu B, Drömann D, Kilinc O. The effect of smoking on COVID-19 symptom severity: Systematic review and meta-analysis. Pulm Med 2020;2020:7590207.

17. Dua K, Surapaneni SN, Kuribayashi S, Hafeezullah M, Shaker R. Protective role of aerodigestive reflexes against aspiration: Study on subjects with impaired and preserved reflexes. Gastroenterology 2011;140:1927-33.

18. World Health Organization (WHO) Coronavirus Disease (Covid-19) Dashboard. 2020. Available at: https:/covid19. who.int/ [Accessed: April 17, 2021]

19. Steele CM, Cichero JA. Physiological factors related to aspiration risk: A systematic review. Dysphagia 2014;29:295-304.

20. Xu Z, Shi L, Wang Y, Zhang J, Huang L, Zhang C, et al. Pathological findings of COVID-19 associated with acute respiratory distress syndrome. Lancet Respir Med $2020 ; 8: 420-2$
21. Cvejic L, Harding R, Churchward T, Turton A, Finlay P, Massey $\mathrm{D}$, et al. Laryngeal penetration and aspiration in individuals with stable COPD. Respirology 2011;16:269-75.

22. Vaira LA, Salzano G, Deiana G, De Riu G. Anosmia and Ageusia: Common findings in COVID-19 patients. Laryngoscope 2020;130:1787.

23. Cirillo N, Bizzoca ME, Lo Muzio E, Cazzolla AP, Lo Muzio L. Gustatory dysfunction in COVID-19 patients: A rapid systematic review on 27,687 cases. Acta Odontol Scand 2021:1-8

24. Santos REA, da Silva MG, do Monte Silva MCB, Barbosa DAM, Gomes ALDV, Galindo LCM, et al. Onset and duration of symptoms of loss of smell/taste in patients with COVID-19: A systematic review. Am J Otolaryngol 2021;42:102889.

25. Baijens LW, Clavé P, Cras P, Ekberg O, Forster A, Kolb GF, et al. European Society for Swallowing Disorders European Union Geriatric Medicine Society white paper: oropharyngeal dysphagia as a geriatric syndrome. Clin Interv Aging 2016;11:1403-28.

26. Lagier A, Melotte E, Poncelet M, Remacle S, Meunier P. Swallowing function after severe COVID-19: early videofluoroscopic findings. Eur Arch Otorhinolaryngol 2021:1-5.

27. Pezdirec M, Strojan P, Boltezar IH. Swallowing disorders after treatment for head and neck cancer. Radiol Oncol 2019;53:225-30.

28. Ticinesi A, Cerundolo N, Parise A, Nouvenne A, Prati B, Guerra A, et al. Delirium in COVID-19: Epidemiology and clinical correlations in a large group of patients admitted to an academic hospital. Aging Clin Exp Res 2020;32:2159-66.

29. Dziewas R, Warnecke T, Zürcher P, Schefold JC. Dysphagia in COVID-19 -multilevel damage to the swallowing network? Eur J Neurol 2020;27:e46-e47.

30. Ceravolo MG, Arienti C, de Sire A, Andrenelli E, Negrini F, Lazzarini SG, et al. Rehabilitation and COVID-19: The Cochrane Rehabilitation 2020 rapid living systematic review. Eur J Phys Rehabil Med 2020;56:642-51.

31. Li L, Li R, Wu Z, Yang X, Zhao M, Liu J, et al. Therapeutic strategies for critically ill patients with COVID-19. Ann Intensive Care 2020;10:45.

32. Virgens IPA, Santana NM, Lima SCVC, Fayh APT. Can COVID-19 be a risk for cachexia for patients during intensive care? Narrative review and nutritional recommendations. Br J Nutr 2020:1-9.

33. O’Keeffe M, Kelly M, O’Herlihy E, O'Toole PW, Kearney PM, Timmons S, et al. Potentially modifiable determinants of malnutrition in older adults: A systematic review. Clin Nutr 2019;38:2477-98.

34. Guy T, Créac'hcadec A, Ricordel C, Salé A, Arnouat B, Bizec JL, et al. High-flow nasal oxygen: A safe, efficient treatment for COVID-19 patients not in an ICU. Eur Respir J. 2020 Nov 12;56(5):2001154.

35. Matthay MA, Aldrich JM, Gotts JE. Treatment for severe acute respiratory distress syndrome from COVID-19. Lancet Respir Med 2020;8:433-4.

36. Mohan R, Mohapatra B. Shedding light on dysphagia associated with COVID-19: The what and why. OTO Open 2020;4:2473974X20934770. 
37. Oomagari M, Fujishima I, Katagiri N, Arizono S, Watanabe $\mathrm{K}$, Ohno $\mathrm{T}$, et al. Swallowing function during high-flow nasal cannula therapy. European Respiratory Journal 2015;46:PA4199.

38. Brodsky MB, Huang M, Shanholtz C, Mendez-Tellez PA, Palmer JB, Colantuoni E, et al. Recovery from dysphagia symptoms after oral endotracheal intubation in acute respiratory distress syndrome survivors. A 5-year longitudinal study. Ann Am Thorac Soc 2017;14:376-83.

39. Padovani AR, Moraes DP, Sassi FC, Andrade CR. Clinical swallowing assessment in intensive care unit. Codas 2013;25:1-7.

40. Frajkova Z, Tedla M, Tedlova E, Suchankova M, Geneid A. Postintubation dysphagia during COVID-19 outbreakcontemporary review. Dysphagia 2020;35:549-57.

41. Brodsky MB, Levy MJ, Jedlanek E, Pandian V, Blackford $B$, Price C, et al. Laryngeal Injury and Upper Airway Symptoms After Oral Endotracheal Intubation With Mechanical Ventilation During Critical Care: A Systematic Review. Crit Care Med 2018;46:2010-7.

42. Bolton L, Mills C, Wallace S, Brady MC; Royal College of Speech and Language Therapists (RCSLT) COVID-19 Advisory Group. Aerosol generating procedures, dysphagia assessment and COVID-19: A rapid review. Int J Lang Commun Disord 2020;55:629-36.

43. Zuercher P, Moret CS, Dziewas R, Schefold JC. Dysphagia in the intensive care unit: Epidemiology, mechanisms, and clinical management. Crit Care 2019;23:103.

44. Macht M, Wimbish T, Clark BJ, Benson AB, Burnham EL, Williams A, et al. Postextubation dysphagia is persistent and associated with poor outcomes in survivors of critical illness. Crit Care 2011;15:R231.

45. Goff D, Patterson J. Eating and drinking with an inflated tracheostomy cuff: A systematic review of the aspiration risk. Int J Lang Commun Disord 2019;54:30-40.

46. Elpern EH, Scott MG, Petro L, Ries MH. Pulmonary aspiration in mechanically ventilated patients with tracheostomies. Chest 1994;105:563-6.

47. Amathieu R, Sauvat S, Reynaud P, Slavov V, Luis D, Dinca $A$, et al. Influence of the cuff pressure on the swallowing reflex in tracheostomized intensive care unit patients. Br J Anaesth 2012;109:578-83.

48. Cooper CA, Urso PP. Gastroesophageal reflux in the intensive care unit patient. Crit Care Nurs Clin North Am 2018;30:123-35.

49. Drinka P. Limiting the impact of antibiotic resistance in nursing homes. J Am Med Dir Assoc 2010;11:537-9.

50. Kölbel CB, Rippel K, Klar H, Singer MV, van Ackern K, Fiedler F. Esophageal motility disorders in critically ill patients: A 24-hour manometric study. Intensive Care Med 2000;26:1421-7.

51. Lima MS, Sassi FC, Medeiros GC, Ritto AP, Andrade CRF. Preliminary results of a clinical study to evaluate the performance and safety of swallowing in critical patients with COVID-19. Clinics (Sao Paulo) 2020;75:e2021.

52. Schindler A, Baijens LWJ, Clave P, Degen B, Duchac $S$, Dziewas R, et al. ESSD commentary on dysphagia management during COVID pandemia. Dysphagia 2020. [Online ahead of print]
53. Malandraki GA, Arkenberg RH, Mitchell SS, Malandraki JB. Telehealth for dysphagia across the life span: Using contemporary evidence and expertise to guide clinical practice during and after COVID-19. Am J Speech Lang Pathol 2021;30:532-50.

54. Miles A, Connor NP, Desai RV, Jadcherla S, Allen J, Brodsky $\mathrm{M}$, et al. Dysphagia care across the continuum: A multidisciplinary dysphagia research society taskforce report of service-delivery during the COVID-19 global pandemic. Dysphagia 2021;36:170-82.

55. South Africa Speech Language Hearing Association. SASLHA's position: Dysphagia Guidelines during COVID19 2020. Available at: 2020. http://www.saslha.co.za [Accessed: April 17, 2021]

56. Speech-Language and Audiology Canada. COVID-19 update: Speech-language pathology services in healthcare settings during the COVID-19 pandemic. 2020. Available at: https://www.sac-oac.ca/news-events/news/covid-19update-speech-language-pathologyservices-healthcaresettings-during-covid [Accessed: April 17, 2021]

57. Royal College of Speech and Language Therapists.RCSLT guidance. 2020. Available at: https://www.rcslt.org/learning/ covid-19/rcsltguidance [Accessed: April 17, 2021]

58. Speech Pathology Australia. Guidance for service delivery. 2020. Available at: https://www.speechpathologyaustralia. org.au/SPAweb/About_us/COVID19_News_and_ Information/COVID19Guidance_for_Service_Delivery/ SPAweb/About_Us/COVID19/Guidance_for_Service_ Delivery.aspx? hkey=fc19a 880 - e7a 8-4246-8631a474fc43d4ae [Accessed: April 17, 2021]

59. Kimura Y, Ueha R, Furukawa T, Oshima F, Fujitani J, Nakajima J, et al. Society of swallowing and dysphagia of Japan: Position statement on dysphagia management during the COVID-19 outbreak. Auris Nasus Larynx 2020;47:715-26.

60. Ku PKM, Holsinger FC, Chan JYK, Yeung ZWC, Chan BYT, Tong MCF, et al. Management of dysphagia in the patient with head and neck cancer during COVID-19 pandemic: Practical strategy. Head Neck 2020;42:1491-6.

61. Brodsky MB, Gilbert RJ. The long-term effects of COVID19 on dysphagia evaluation and treatment. Arch Phys Med Rehabil 2020;101:1662-4.

62. Araújo BCL, Domenis DR, Ferreira THP, Merelles CLA, Lima TRCM. COVID-19 and dysphagia: practical guide to safe hospital care - number 1. Audiol Commun Res 2020;25:e2384.

63. Carda S, Invernizzi M, Bavikatte G, Bensmail D, Bianchi F, Deltombe T, et al. COVID-19 pandemic. What should Physical and Rehabilitation Medicine specialists do? A clinician's perspective. Eur J Phys Rehabil Med 2020;56:515-24.

64. Freeman-Sanderson A, Ward EC, Miles A, de Pedro Netto I, Duncan S, Inamoto Y, et al. A consensus statement for the management and rehabilitation of communication and swallowing function in the ICU: A global response to COVID-19. Arch Phys Med Rehabil 2020:S00039993(20)31210-7.

65. Fernández-Ruiz VE, Paredes-Ibáñez R, Armero-Barranco D, Sánchez-Romera JF, Ferrer M. Analysis of quality of life and nutritional status in elderly patients with dysphagia in order to prevent hospital admissions in a COVID-19 pandemic. Life (Basel) 2020;11:22. 